\title{
Short communication: Characterization of Salmonella phages from dairy calves on farms with history of diarrhea
}

\author{
Fernando Dueñas, ${ }^{*}$ Dácil Rivera, ${ }^{*}$ Viviana Toledo, ${ }^{*}$ Rodolfo Tardone, ${ }^{*}$ Luis P. Hervé-Claude, $†$ \\ Christopher Hamilton-West, $\ddagger$ and Andrea I. Moreno Switt*1 \\ *Escuela de Medicina Veterinaria, Facultad de Ecología y Recursos Naturales, Universidad Andres Bello, Santiago, Chile 8320000 \\ †Departamento de Ciencias Clínicas, and \\ ‡Departamento Medicina Preventiva, Facultad de Ciencias Veterinarias y Pecuarias, Universidad de Chile, Santiago, Chile 8820808
}

\begin{abstract}
Salmonella enterica can cause disease and mortality in calves. This pathogen is also a zoonosis that can be transmitted by animal contact or by food. The prevalence of Salmonella in dairy farms has been reported to range from 0 to $64 \%$, and, due to the diversity of Salmonella serovars that can be circulating, Salmonella is an important concern for dairy production. Bacteriophages that infect Salmonella have been documented to be abundant and widely distributed in the dairy environment. The current study investigated the diversity of Salmonella serovars and Salmonella phages in 8 dairy farms with a history of diarrhea in southern Chile. A total of 160 samples from sick calves, healthy calves, and the environment were analyzed for Salmonella and phage. Isolated phages were characterized and classified by their host range using a panel of 26 Salmonella isolates representing 23 serovars. Host ranges were classified according to lysis profiles (LP) and their spatial distribution was mapped. Salmonella-infecting phages were identified, but none of the 160 samples were positive for Salmonella. A total of 45 phage isolates were obtained from sick calves (11), healthy calves (16), or the environment (18). According to their host range, 19 LP were identified, with LP1 being the most common on all 8 farms; LP1 represents phages that only lyse serogroup D Salmonella. The identification of Salmonella phages but not Salmonella in the same samples could suggest that these phages are controlling Salmonella in these farms.
\end{abstract}

Key words: Salmonella, bacteriophage, dairy calf

\section{Short Communication}

Salmonella is one of the most common foodborne pathogens in the world, causing an estimated 230,000

Received June 6, 2016.

Accepted November 11, 2016.

${ }^{1}$ Corresponding author: andrea.moreno@unab.cl deaths yearly (Havelaar et al., 2015). As a zoonotic agent, Salmonella is also transmitted by animal contact (Hoelzer et al., 2011a). In cattle, serovars of Salmonella enterica ssp. enterica have been reported to cause clinical illness (e.g., Salmonella Newport, Salmonella Dublin) and to colonize asymptomatic carriers (e.g., Salmonella Cerro; Rodriguez-Rivera et al., 2014). Prevalence of Salmonella in dairy cattle varies; one study of bovine clinical outbreaks in the United States reported a prevalence of fecal shedding ranging from 0 to $53 \%$ (Cummings et al., 2010). A recent study reported Salmonella isolates in up to $64 \%$ of fecal samples from dairy farms in Texas (Rodriguez-Rivera et al., 2016). In Chile, there is a dearth of knowledge about Salmonella in dairy farms. One thesis conducted in 2004 in southern Chile reported Salmonella in $14 \%$ of sampled dairy farms $(4 / 28)$ (Barrientos, 2005). There are no contemporary publications on the presence of Salmonella in dairy farms in Chile, except for one report from surveillance conducted by the Chilean Government that reported the presence of abortions caused by Salmonella Dublin in Chile in 2013 (http://www.sag.cl/sites/default/files/ situacion_sanitaria_animal_2013_0.pdf).

The most common serovars of Salmonella reported in cattle in different parts of the world include Newport, Cerro, Typhimurium, Kentucky, and Dublin (Warnick et al., 2006; Hoelzer et al., 2011b; Rodriguez-Rivera et al., 2014, 2016). Although some of these serovars, such as Salmonella Typhimurium, represent a serovar widely distributed in different hosts, other serovars, such as Salmonella Dublin, are considered to be better adapted to the bovine host (Nielsen, 2013). Salmonella Dublin isolated from cattle has been reported in several studies (Veling et al., 2002; Warnick et al., 2006; Hoelzer et al., 2011b; Nielsen and Dohoo, 2012) and is the serovar most commonly reported in clinical cases of calves, which are highly susceptible between 3 and 6 wk of age (Veling et al., 2002).

The gold standard to detect the presence of Salmonella in cattle is culture of bacteria from feces; however, studies have reported that serology has better herd-level 
sensitivity than culturing (Veling et al., 2002). This difference is due, in large part, to the fact that Salmonella shedding in the feces is transient, varies over the shedding period, and is affected by antimicrobial treatments (Veling et al., 2002; Nielsen, 2013).

Salmonella bacteriophages appear to be widespread on dairy farms, and the diversity of these phages could reveal interactions between Salmonella serovars and specific bacteriophages (Moreno Switt et al., 2013). Salmonella phages have been found to be very abundant (up to $97 \%$ prevalence) and widely distributed in animal feces, wastewater, and the environment of food animals (Moreno Switt et al., 2013; Pérez Pulido et al., 2016). In addition, studies of Salmonella phage host range appear to indicate Salmonella serovar specificity. For example, farms with a high prevalence of Salmonella Cerro were found to have a predominance of phages specific to Salmonella Cerro (Moreno Switt et al., 2013). Another study investigated the host range of Salmonella phages isolated from dairy farms in the United States and Thailand and found that US phages showed a narrow host range, whereas Thai phages showed a wide phage host range (Wongsuntornpoj et al., 2014). In this current study, we hypothesized that Salmonella phages obtained from calves would have a wide host range, lysing a variety of serovars including Salmonella isolates from calves in the same dairy. We characterized Salmonella phage dynamics in sick and healthy dairy calves to improve our understanding of the role of phages in controlling Salmonella in calves.

Samples were collected from 8 farms located in the Los Ríos and Los Lagos regions of southern Chile. Farms with calves with symptoms of diarrhea were selected based on a target sampling methodology that enrolled farmers willing to take part in a Salmonella characterization study. Samples were taken from 10 sick calves, 5 healthy calves, and 5 environmental samples from each farm. Sick calves were selected based on clinical signs and evidence of diarrhea, and healthy calves and environmental samples were collected based on simple random sampling after enumerating all calves and pens using a random number generator in a handheld device. Environmental samples (e.g., holding area, manure storage, and animal beds) were collected in $60-\mathrm{mL}$ sterile containers, using a protocol previously described (Rodriguez-Rivera et al., 2016). Fecal samples were collected from each animal per rectum after a gentle massage of the area. Overall, 160 samples were collected (80 from sick calves, 40 from healthy calves, and 40 from the environment); samples were kept at $4^{\circ} \mathrm{C}$ and transferred to the laboratory at Universidad Andres Bello (Santiago, Chile) for processing within $24 \mathrm{~h}$.

All samples were cultured for Salmonella. Briefly, $10 \mathrm{~g}$ of each sample was enriched in $90 \mathrm{~mL}$ of peptone water (Becton-Dickinson, Franklin Lakes, NJ) and incubated at $37^{\circ} \mathrm{C}$ overnight. Two subsets were then incubated: one set of $1 \mathrm{~mL}$ of sample in $9 \mathrm{~mL}$ of tetrathionate broth (Becton-Dickinson) with iodine and the other set of $100 \mu \mathrm{L}$ of sample in $9.9 \mathrm{~mL}$ of Rappaport Vassiliadis broth (Becton-Dickinson) with novobiocin $(20 \mu \mathrm{g} / \mathrm{mL})$. Finally, aliquots of $100 \mu \mathrm{L}$ were inoculated onto XLT4 agar with XLT4 supplement (xylose lactose tergitol; Becton-Dickinson). Suspect colonies were confirmed by invA PCR (Kim et al., 2007). To validate the isolation methods, random samples were processed in the Laboratory of Food at Universidad Austral (Campus Isla Teja, Valdivia, Chile), which is ISO-certified for Salmonella detection.

To isolate phages, equal proportions of all samples from a single source on each farm were combined; specifically, samples from sick calves were pooled, samples from healthy calves were pooled, and environmental samples were pooled, forming 3 pooled samples per farm. These pooled samples were used to isolate and purify Salmonella phages, as previously described (Moreno Switt et al., 2013). Four Salmonella strains,

Table 1. Summary of phages detected and purified from dairy calves in this study

\begin{tabular}{|c|c|c|c|c|c|c|}
\hline $\begin{array}{l}\text { Farm } \\
\text { ID }\end{array}$ & Sampling date & $\begin{array}{c}\text { Total isolated } \\
\text { phages }\end{array}$ & \multicolumn{4}{|c|}{ Number of Salmonella phages isolated ${ }^{1}$} \\
\hline 2 & April 28, 2015 & 8 & $3(1 / 1 / 1)$ & $4(0 / 2 / 2)$ & $1(0 / 0 / 1)$ & 0 \\
\hline 3 & April 29, 2015 & 6 & $3(1 / 1 / 1)$ & 0 & $3(1 / 1 / 1)$ & 0 \\
\hline 4 & May 28, 2015 & 3 & $3(1 / 2 / 0)$ & 0 & 0 & 0 \\
\hline 7 & May 29,2015 & 6 & $4(0 / 2 / 2)$ & $2(0 / 0 / 2)$ & 0 & 0 \\
\hline 8 & August 26, 2015 & 4 & $4(0 / 2 / 2)$ & 0 & 0 & 0 \\
\hline Total & & 45 & 29 & 9 & 4 & 3 \\
\hline
\end{tabular}

${ }^{1}$ Number of phages displaying clear plaques that were identified and purified in the strain representing the 4 Salmonella serovars (numbers in parentheses indicate samples from sick calves/healthy calves/environment). FSL = Food Safety Laboratory at Cornell University (Ithaca, NY). 
previously characterized by the Food Safety Laboratory (FSL) at Cornell University (Ithaca, NY), were used as host for phage isolation: Infantis (FSL S5-506), Heidelberg (FSL S5-455), Typhimurium (FSL S5-370), and Enteritidis (FSL S5-371). The host range of the isolated phages was characterized using a panel of 26 Salmonella strains using previously described protocols (Vongkamjan et al., 2012; Moreno Switt et al., 2013). In addition, one isolate of Escherichia coli and one isolate of Pseudomonas spp. were added. Host ranges were

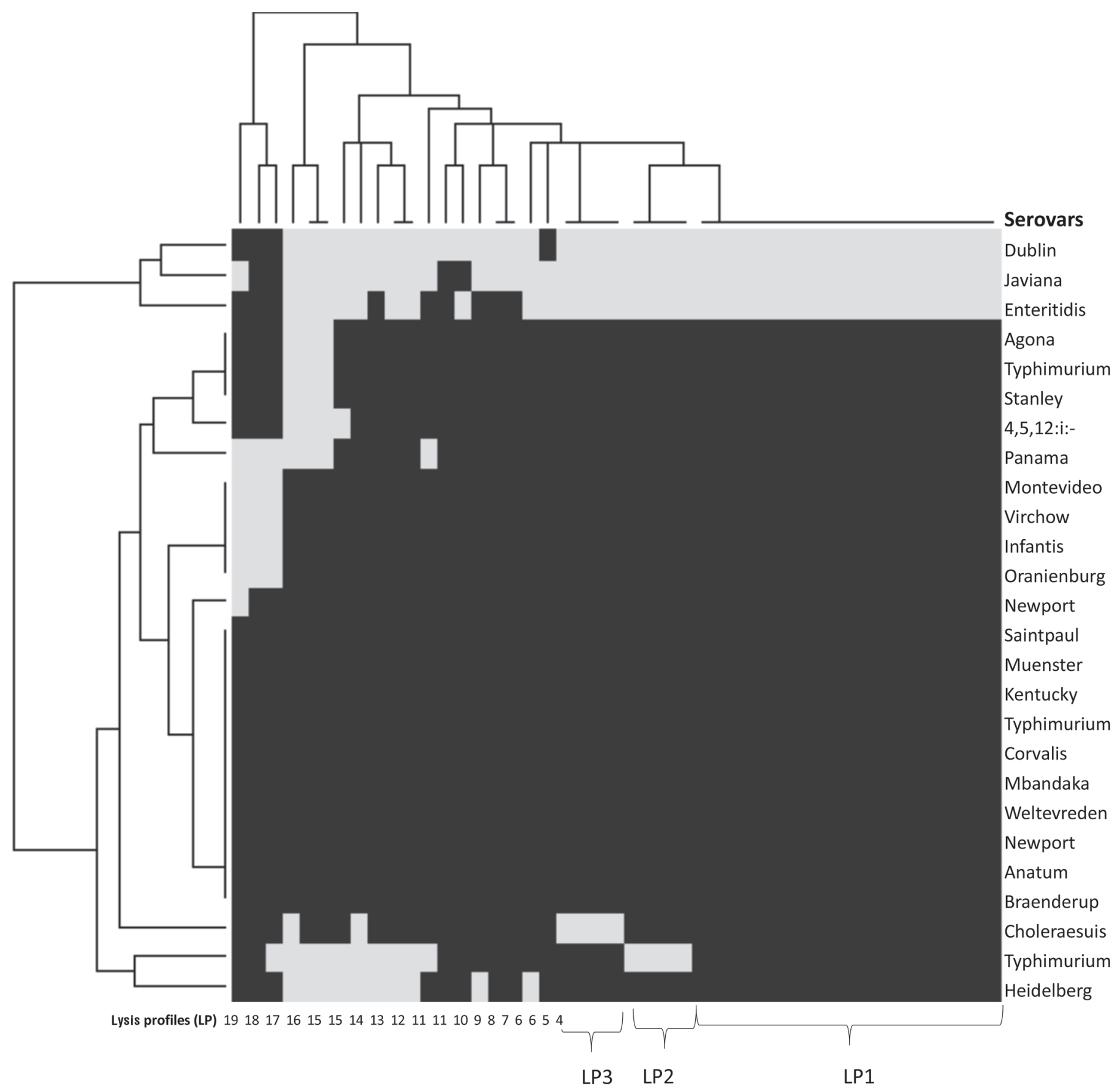

Figure 1. Heatmap representing phage relatedness according to the phage lysis profiles. A hierarchical clustering was conducted using the Ward's method of binary distance with the software R (version 2.10.0; R Development Core Team, Vienna, Austria; http://www.R-project.org). In the $\mathrm{x}$-axis are the lysis profiles (LP) and in the y-axis are the serovars used to characterize the phages identified in this study. Gray represents lysis and black represents no lysis. 
classified by lysis profiles (LP). Results were mapped using spatial software (ArcGIs v.10, Esri, Redlands, CA).

This study searched for Salmonella and Salmonella phages in dairy farms with history of calves with diarrhea; Salmonella was not identified in any of the 160 samples tested. Importantly, in the sampled farms, antimicrobial treatment is generally used to treat diarrhea, but treatments are poorly recorded; therefore, it is possible that these antimicrobial treatments inhibited Salmonella growth, explaining our results.

In this study, we identified an abundance of bacteriophages in sick calves (11 phages), healthy calves (16 phages), and the environment (18 phages) of all 8 sampled dairy farms (Table 1), with significantly more bacteriophages being isolated from healthy calves than from sick calves $\left(\chi^{2}=11.933, P<0.05\right)$. Previous studies have also reported an abundance of phages in dairy farms in different parts of the world with varied productions systems (i.e., intensive or backyard; Moreno Switt et al., 2013; Wongsuntornpoj et al., 2014).

This study detected and purified 45 phages; the majority were isolated with the Salmonella Enteritidis host (64.4\%), followed by Salmonella Heidelberg (20\%), Salmonella Typhimurium (8.9\%), and Salmonella Infantis $(6.7 \%)$ (Table 1). In previous studies in the United States and Thailand, phages from dairy farms were mostly isolated using serovars Weltevreden, Newport, Enteritidis, Typhimurium, and Cerro (Moreno Switt et al., 2013; Wongsuntornpoj et al., 2014). Although a previous study reported that a high prevalence of Salmonella Cerro was associated with the isolation of Salmonella phages infecting this serovar, Salmonella Cerro was not detected in this study. Salmonella Cerro has been described as being adapted to the bovine intestine (Hoelzer et al., 2011b; Rodriguez-Rivera et al., 2014). Our findings, along with previous reports, indicate an association between Salmonella serovars and phages capable of infecting these serovars on the same farm.

The host range characterization identified 19 LP (Figure 1; Supplemental Table S1; https://doi.org/10.3168/ jds.2016-11569) that were widely distributed among the farms (Supplemental Figure S1; https://doi. org/10.3168/jds.2016-11569). Among all LP, LP1 was the most frequent and widely distributed (18 phages from all 8 farms); these profiles included phages that lysed Salmonella Enteritidis, Salmonella Javiana, and Salmonella Dublin, all serogroup D Salmonella. Importantly, LP1 was found in farms up to 120 miles apart (Supplemental Figure S1). In addition, these 3 serovars were found to be the most susceptible to phage lysis in this study (Figure 1). Previously, our group identified serogroup D-specific phages in other regions of Chile, suggesting a very wide distribution of phages infecting serogroup D Salmonella (our unpublished data).

To determine if the isolated phages could infect other bacteria, we tested whether isolated phages could lyse isolates of E. coli and Pseudomonas spp. None of the phages lysed these bacteria. Although some Salmonella phages have been reported to lyse E. coli (Moreno Switt et al., 2013), phages obtained from calves in this study appear to be specific to Salmonella. Overall, results from this study indicate that Salmonella phages are abundant and widely distributed in sick and healthy dairy calves; it is tempting, therefore, to speculate that these widely distributed phages might be controlling Salmonella in the feces of these animals.

\section{ACKNOWLEDGMENTS}

This study was funded, in part, by FONDECYT 11140108 to AIMS (Santiago, Chile) and Proyecto UInicia VID Dr. Luis Hervé 1210170119102106 (Universidad de Chile, Santiago, Chile). The funding sources had no role in the study design; collection, analysis, and interpretation of data, writing of the report, or the decision to submit the manuscript for publication. We thank Randall Singer (School of Public Health, University of Minnesota, St. Paul) for editing the manuscript.

\section{REFERENCES}

Barrientos, L. 2005. Detección de Salmonella spp. en fecas de terneros de predios lecheros de tamaño superior a 100 hecáreas en la comuna de Paillaco. Universidad Austral de Chile, Campus Isla Teja, Valdivia, Chile.

Cummings, K. J., L. D. Warnick, M. Elton, Y. T. Gröhn, P. L. McDonough, and J. D. Siler. 2010. The effect of clinical outbreaks of salmonellosis on the prevalence of fecal Salmonella shedding among dairy cattle in New York. Foodborne Pathog. Dis. 7:815823. https://doi.org/10.1089/fpd.2009.0481.

Havelaar, A. H., M. D. Kirk, P. R. Torgerson, H. J. Gibb, T. Hald, R. J. Lake, N. Praet, D. C. Bellinger, N. R. de Silva, N. Gargouri, N. Speybroeck, A. Cawthorne, C. Mathers, C. Stein, F. J. Angulo, and B. Devleesschauwer. 2015. World Health Organization global estimates and regional comparisons of the burden of foodborne disease in 2010. PLoS Med. 12:e1001923. https://doi.org/10.1371/ journal.pmed.1001923.

Hoelzer, K., K. J. Cummings, E. M. Wright, L. D. Rodriguez-Rivera, S. E. Roof, A. I. M. Switt, N. Dumas, T. Root, D. J. Schoonmaker-Bopp, Y. T. Grohn, J. D. Siler, L. D. Warnick, D. D. Hancock, M. A. Davis, and M. Wiedmann. 2011b. Salmonella Cerro isolated over the past twenty years from various sources in the US represent a single predominant pulsed-field gel electrophoresis type. Vet. Microbiol. 150:389-393. https://doi.org/10.1016/j. vetmic.2011.01.026.

Hoelzer, K., A. I. Moreno Switt, and M. Wiedmann. 2011a. Animal contact as a source of human non-typhoidal salmonellosis. Vet. Res. 42:34. https://doi.org/10.1186/1297-9716-42-34.

Kim, J. S., G. G. Lee, J. S. Park, Y. H. Jung, H. S. Kwak, S. B. Kim, Y. S. Nam, and S. T. Kwon. 2007. A novel multiplex PCR assay for rapid and simultaneous detection of five pathogenic bacteria: Escherichia coli O157:H7, Salmonella, Staphylococcus aureus, Lis- 
teria monocytogenes, and Vibrio parahaemolyticus. J. Food Prot. 70:1656-1662.

Moreno Switt, A. I., H. C. den Bakker, K. Vongkamjan, K. Hoelzer, L. D. Warnick, K. J. Cummings, and M. Wiedmann. 2013. Salmonella bacteriophage diversity reflects host diversity on dairy farms. Food Microbiol. 36:275-285.

Nielsen, L. R. 2013. Review of pathogenesis and diagnostic methods of immediate relevance for epidemiology and control of Salmonella Dublin in cattle. Vet. Microbiol. 162:1-9. https://doi. org/10.1016/j.vetmic.2012.08.003.

Nielsen, L. R., and I. Dohoo. 2012. Survival analysis of factors af fecting incidence risk of Salmonella Dublin in Danish dairy herds during a 7-year surveillance period. Prev. Vet. Med. 107:160-169. https://doi.org/10.1016/j.prevetmed.2012.06.002.

Pérez Pulido, R., M. J. Grande Burgos, A. Gálvez, and R. Lucas López. 2016. Application of bacteriophages in post-harvest control of human pathogenic and food spoiling bacteria. Crit. Rev. Biotechnol. 36:851-861. https://doi.org/10.3109/07388551.2015.1 049935 .

Rodriguez-Rivera, L. D., K. J. Cummings, G. H. Loneragan, S. C Rankin, D. L. Hanson, W. M. Leone, and T. S. Edrington. 2016. Salmonella prevalence and antimicrobial susceptibility among dairy farm environmental samples collected in Texas. Foodborne Pathog. Dis. https://doi.org/10.1089/fpd.2015.2037.

Rodriguez-Rivera, L. D., E. M. Wright, J. D. Siler, M. Elton, K. J. Cummings, L. D. Warnick, and M. Wiedmann. 2014. Subtype analysis of Salmonella isolated from subclinically infected dairy cattle and dairy farm environments reveals the presence of both human- and bovine-associated subtypes. Vet. Microbiol. 170:307316. https://doi.org/10.1016/j.vetmic.2014.02.013.

Veling, J., H. Barkema, J. van der Schans, F. van Zijderveld, and J. Verhoeff. 2002. Herd-level diagnosis for Salmonella enterica ssp. enterica serovar Dublin infection in bovine dairy herds. Prev. Vet. Med. 53:31-42. https://doi.org/10.1016/S0167-5877(01)00276-8.

Vongkamjan, K. A. M. Switt, H. C. den Bakker, E. D. Fortes, and M. Wiedmann. 2012. Silage collected from dairy farms harbors an abundance of listeriaphages with considerable host range and genome size diversity. Appl. Environ. Microbiol. 78:8666-8675. https://doi.org/10.1128/AEM.01859-12.

Warnick, L. D., L. R. Nielsen, J. Nielsen, and M. Greiner. 2006. Simulation model estimates of test accuracy and predictive values for the Danish Salmonella surveillance program in dairy herds. Prev. Vet. Med. 77:284-303. https://doi.org/10.1016/j prevetmed.2006.08.001.

Wongsuntornpoj, S., A. I. Moreno Switt, P. Bergholz, M. Wiedma$\mathrm{nn}$, and S. Chaturongakul. 2014. Salmonella phages isolated from dairy farms in Thailand show wider host range than a comparable set of phages isolated from U.S. dairy farms. Vet. Microbiol. 172:345-352. https://doi.org/10.1016/j.vetmic.2014.05.023. 